\title{
Acupoint Autohemotherapy Attenuates DNCB- induced Atopic Dermatitis Lesions by Regulating Th1/Th2 Cytokine Balance in BALB/c Mice
}

\section{Zhiwen Zeng}

Southern Medical University https://orcid.org/0000-0002-9530-6701

Jinquan Huang

Southern Medical University

\section{Yong Chen}

Shenzhen People's Hospital

Xiao Yu

Southern Medical University

Wei Zhu

Guangzhou Center for Disease Control and Prevention

Dongshu Zhang ( $\nabla$ nyzds@sina.com )

Southern Medical University https://orcid.org/0000-0002-8390-7235

\section{Research}

Keywords: Acupoint autohemotherapy, Atopic dermatitis, Th1/Th2, IgE

Posted Date: November 23rd, 2021

DOI: https://doi.org/10.21203/rs.3.rs-1081783/v1

License: () (1) This work is licensed under a Creative Commons Attribution 4.0 International License. Read Full License 


\section{Abstract \\ Objective}

Acupoint autohemotherapy (A-AHT) is considered an effective therapy for atopic dermatitis (AD) with few side-effects. Previous experiments showed the treatment had the potential to regulate $T$ helper (Th) 1 and Th2 cytokines, like interferon (IFN)- gamma and interleukin (IL)- 4 . This study focuses on the effects of AAHT on the AD-like skin lesions through regulating Th1/Th2 immune responses.

\section{Methods}

The treatments of A-AHT, sham acupoint autohemotherapy and acupoint injection of normal saline were administered in the AD mice once every other day for 4 weeks. The total immunoglobulin (Ig) $E$, IL-4 and IFN- $y$ cytokine levels in the serum were examined after animal sacrifice. Th1/Th2 expression was analyzed in murine spleen cells via flow cytometry and immunohistochemical analysis of GATA-3 and Tbet in skin lesions were further assessed.

\section{Results}

Either type of repeated autologous whole blood (AWB) injection (into acupoint or sham acupoint) reduced the severity of AD-like symptoms and level of serum IgE. All of the three treatments had the similar inhibitory effect on levels of IL-4 and upregulation on the ratio of IFN- $\mathrm{Y} / \mathrm{IL}-4$, while differed on Th1/Th2 ratio as A-AHT regulates the body's Th1/Th2 shift. This treatment also increased the related transcription factors T-bet expression, and upregulated T-bet/GATA3 ratio compared with the DNCB group. These differences were significant only in A-AHT group.

\section{Conclusion}

A-AHT effectively reduces AD symptoms and serum IgE levels in a mouse model and may act by regulating Th1/Th2 immune responses.

\section{Introduction}

Atopic dermatitis (AD) is a chronic and relapsing form of atopic skin inflammation, characterized by erythema, dryness and recurrent pruritus, which is driven by a combination of penetrating allergens (impaired skin barrier), abnormal T cell sub populations [1], higher levels of total serum IgE and inflammatory cells such as mast cells all working together to induce an allergic response [2]. The overactive immune system, especially the abnormal activation of Thelper (Th)2 cells, is related to the progression of the disease, which contributes to a predominant Th2 response in the acute lesions of $A D$, and an imbalance between Th1 and Th2 responses [3-4]. The increased expression of classic Th2 
cytokines, such as IL-4, IL-5 and IL-31, mediate the secretion of immunoglobulin E (IgE). Furthermore, high IgE activates mast cells by combining with the IgE receptor FcERI, which sits on the surface of mast cells. Activated mast cells release histamine and other biologically active products, triggering allergic inflammation symptoms [5]. Studies have shown that the immune response can be significantly suppressed by regulating the balance between Th1 and Th2 by shifting it to Th1 dominance, which indicates that this is an effective strategy for the treatment of $A D[6-7]$.

Autohemotherapy (or autologous blood injection), is a treatment using repeated intramuscular injections of autologous whole blood (AWB) to cure certain diseases. It has been used in European countries since the end of 20th century [8]. Recent clinical studies shown that autohemotherapy might be an effective and potentially disease-modifying or curable treatment in patients with $\mathrm{AE}$ [9]. In China, autohemotherapy has attracted the interest from many traditional Chinese medicine (TCM) physicians and researchers. Based on the TCM theory, a new treatment was created by combining the autohemotherapy with the acupuncture therapy, and named as acupoints autohemotherapy (A-AHT) or as AWB acupoints injection [10]. A-AHT is considered an effective therapy with few side effects, however, the mechanism is still unclear. In this study, we discussed the effects of A-AHT on AD mice and orientated the molecular mechanism towards whether it can rebalance Th1/Th2 by regulating the Th2-related GATA-3 and Th1related T-bet.

\section{Materials And Methods}

\section{Animals}

All animal experiments were performed according to the Health Guide for the Care and Use of Laboratory Animals [11] and were approved by the medical ethics committee of Southern Medical University. A total of 30 female BALB/c mice, aged 6 weeks, were purchased from Experimental animal center of Southern Medical University (Guangzhou, China, No. SCXK 2016-0041) and housed indoors under SPF conditions. After one week of acclimatization, the mice were randomly divided into five groups ( $n=6$ per group) as follows: (1) a normal control group (NC), (2) a DNCB (1-chloro-2,4-dinitrobenzene, Sigma, St. Louis, MO, USA)-sensitized and challenged group (DNCB), (3) a DNCB-sensitized and challenged group with acupoint autohemotherapy treatment (A-AHT), (4) a DNCB-sensitized and challenged group with sham acupoint autohemotherapy treatment (S-AHT), (5) a DNCB-sensitized and challenged group with acupoint injection of normal saline (A-NS).

\section{$A D$ induction in $B A L B / c$ mice}

Establishment of the AD model and timing of the treatments are described in Figure $1 \mathrm{~A}$. For the first induction of $A D$ in the BALB/c mice, the backs of the mice were shaved and challenged with $200 \mu \mathrm{L}$ of $0.5 \%$ DNCB in acetone/olive oil (3:1) for 4 days (day 1 - day 4). After 7 days from the first induction, $0.5 \%$ DNCB was painted onto the backs of the mice every other day for 28 days (day 8 - day 35) [12].

\section{Treatment}


The mice received treatment once every other day since the 8th day of the experiment. The animals were treated after sodium pentobarbital $(1 \%, 50 \mathrm{mg} / \mathrm{kg}, \mathrm{I.P})$ anesthesia and shown no evident signs of distress. Except for the NC group, total $0.2 \mathrm{ml}$ of blood was withdrawn from the facial vein using a $1 \mathrm{ml}$ syringe assorted with $5 \#$ needle in each treatment. For the A-AHT group, $0.05 \mathrm{ml}$ of AWB in each acupoint was injected into bilateral Zusanli (ST36) and Quchi (IL11) [13, 14]. The whole procedure was within one minute. For the S-AHT group, $0.05 \mathrm{ml}$ of AWB was injected into each sham-acupoint ( $5 \mathrm{~mm}$ lateral to ST36 and IL 11). For the A-NS group, $0.05 \mathrm{ml}$ of $0.9 \%$ normal saline was injected into bilateral ST 36 and IL11. The Zusanli (ST36) is located near the knee joint of the hind limb, $1.5 \mathrm{~mm}$ away from the distal anterior tibial tubercle. The sham point localized $5 \mathrm{~mm}$ above the Zusanli (ST36) [15]. The Quchi (LI11) located at the lateral end of the cubital crease. The sham point of Quchi (LI11) was located at the midpoint of the acromial part of the deltoid muscle [16]. The location of all acupoints is shown in Figure 1B. During treatment, the mice of NC group and DNCB group were hold in the hand for about one minute to exclude the stress response. On day 35 , all animals were sacrificed.

\section{Evaluation of dermatitis severity}

Dermatitis severity was determined using the SCORAD scoring standard proposed by the European AD research group (ETFAD) [17]. The symptom scores were measured every morning after the DNCB challenge from day 7 to 35 , within 30 min of administration of DNCB. Development of erythema, papule /oedema, oozing/crust, exfoliation, dryness and lichenification were scored as follows: 0 (none), 1 (mild, $<20 \%$ ), 2 (moderate, $20-60 \%$ ), and 3 (severe, $>60 \%$ ). A total score was defined as the sum of obtained scores (SCORAD index, scale 0-18).

\section{Histological analysis}

The dorsal skin tissue of the mice was fixed in $4 \%$ neutral buffered formalin (NBF) for $24 \mathrm{~h}$. The tissue was then embedded in paraffin, sliced into $4-\mu \mathrm{m}$-thick sections, and stained with hematoxylin and eosin $(H \& E)$. The sections were reviewed by two trained pathologist to assess the tissues. The number of mast cells was counted following toluidine blue (TB) staining. All sections were examined by light microscopy (Nikon, Eclipse Ci-L, Japan), using 5 fields at 100-fold magnification.

\section{Immunohistochemistry}

Prepared sections of dorsal skin were immersed in $0.01 \mathrm{M}, \mathrm{pH} 6.0$ citrate buffer ( $30 \mathrm{~min}$ ) in a water bath maintained at $100^{\circ} \mathrm{C}$ for antigen retrieval. The tissue sections are placed in $3 \%$ hydrogen peroxide $(25 \mathrm{~min}$ at room temperature in darkness). After washing 3 times in PBS, 3\% BSA was added (30 min at room temperature) to block non-specific binding sites. The T-bet/GATA3 antibody (Proteintech, catalog no. 13700-1-AP/66400-1-Ig, U. S. A) prepared with PBS (PH7.4) in 1:200 was added to the sections, at $4^{\circ} \mathrm{C}$ overnight in a wet box followed by staining with a DAB chromogenic agent kit (Servicebio, catalog no. G1211, China), then stained with freshly prepared diaminobenzidine solution and counterstained with hematoxylin. Sections were dehydrated with a graded series of alcohol, vitrified by dimethylbenzene and covered with neutral gum. Finally, a microscope (Nikon, E100, Japan, 200 magnification) was used to 
analyze the relevant parts of the sample. Immunohistochemistry (IHC) staining of T-bet and GATA-3 were also assessed by semi-quantitative analysis using ImageJ software. The results were shown by average optical density (AOD).

\section{Flow cytometry}

The spleens tissues of mice were minced and filtered with a $70 \mu \mathrm{m}$ sieve to obtain a single cell suspension. The red Blood Cell Lysis Buffer (Pythonbio, catalog no. AAPR27, China) was added to the single cell suspension for $10 \mathrm{~min}$ at $4^{\circ} \mathrm{C}$. After centrifugation and resuspension, adjust the cell concentration to $1 \times 10^{\wedge} 6 / \mathrm{mL}$. Then, the mouse Th1/Th2 staining kit (MultiSciences, catalog no. KTH201, China) was used for staining, following the manufacturer's protocol. Th1 cells were assessed using antiCD3 $\varepsilon$ FITC, anti-CD4 PerCP-Cy5.5 and anti-IFN-y PE. Th2 cells were assessed using anti-CD3 $\varepsilon$ FITC, antiCD4 PerCP-Cy5.5 and anti-IL-4 APC. Samples were analyzed using a flow cytometer (BD Biosciences, BD FACSCalibur, USA) and CellQuest Pro 5.1 software (BD Biosciences, USA). The spleen index was calculated as: Spleen index $=($ spleen weight/body weight $) \times 100 \%$.

\section{Enzyme linked immunosorbent assay (ELISA)}

Serum samples were obtained from the blood of inferior vena cava by centrifugation (3000rpm, $10 \mathrm{~min}$ ) and stored at $-80^{\circ} \mathrm{C}$. Levels of total serum IgE, IFN- $\gamma$ and IL-4 were measured using a mouse enzyme linked immunosorbent assay (ELISA) kit (MultiSciences, catalog no. EK275, EK271, EK282/4, EK280/3, EK204/2, China), following the manufacturer's protocol.

\section{Statistics analysis}

Results of data are expressed as means \pm standard errors of the means (SEMs). One-way analysis of variance (ANOVA) was used for multiple comparisons, followed by least-significant difference post hoc test (SPSS, Version 24.0). Results represented as significance based on a value of $P<0.05$.

\section{Results}

\section{Acupoint autohemotherapy attenuates DNCB-Induced AD-Like symptoms}

To investigate the effects of A-AHT on the DNCB-induced AD-like symptoms in the Balb/c mice, we assessed the dermatitis severity of their dorsal skin. Representative images of the dorsal skin from each group are shown in Figure 2A. In addition, erythema, papule/oedema, oozing/crust, excoriation, dryness and lichenification of the skin area in the experimental mice were recorded on days 7, 14, 21, 28 and 35. The dermatitis scores of each group were calculated and are plotted in Figure 2B. The results indicate that DNCB can remarkably induce skin dysfunction over time, whereas A-AHT and S-AHT can significantly alleviate these symptoms.

\section{Acupoint autohemotherapy inhibits epidermal hyperplasia and infiltration of mast cells in AD lesion}


To further investigate the therapeutic effects of A-AHT on AD-like dorsal lesions, we examined the H\&E and TB staining sections under higher magnification to evaluate epidermal hyperplasia, dermal thickness and infiltration of mast cells in the dorsal skin tissue (Figure 3A, B). After 28 days of treatment, the A-AHT group and S-AHT group had significantly less epidermal thickening than the DNCB group did (Figure 3C). The dermal thickness and number of infiltrated mast cells (Figure. 3D, E) were significantly decreased in A-AHT group and A-NS group, compared to the DNCB group. These results indicate that A-AHT have significant attenuating effects on $A D$.

\section{Acupoint autohemotherapy shift the ratio of Th1/Th2 in murine spleen}

In order to assess the effect of A-AHT on Th1/Th2 ratio of lymphocytes (LCs) in spleen. We detected cell numbers expressing of Th1/Th2 by Flow Cytometry. Results as shown in Figure 4 indicated that the ratio of Th1/Th2 was decreased in DNCB group and increased in A-AHT group. Furthermore, the spleen index was increased in DNCB group and decreased in A-AHT group, S-AHT group and A-NS group.

\section{A-AHT down-regulated IgE level in serum and the levels of inflammatory cytokines in model mice}

To evaluate the effects of A-AHT on immune responses in the AD mouse model, we measured the serum levels of IgE, IL-4 and IFN- $\gamma$. We found that IgE level in serum was increased in DNCB group. The increase was suppressed significantly in groups treated with A-AHT and S-AHT. The same as above, the levels of IL-4 and IFN- $\gamma$ was increased in DNCB group and decreased in groups treated with A-AHT and S-AHT. However, it showed a similar efficacy between the two treatment groups (Figure $5 \mathrm{~A}-\mathrm{C}$ ).

\section{Acupoint autohemotherapy regulates the expression of T-bet and GATA3 in DNCB-Induced Balb/c mice}

The transcription factors T-bet and GATA3 are crucial regulators of the differentiation of precursor cells into Th1 cells, which produce IFN-y, and Th2 cells, which produce IL-4. Therefore, we examined the effects of A-AHT treatment on T-bet and GATA3 expression in the skin tissue of mice with AD. As shown in Figure 6A, mice in NS group basically expressed T-bet and GATA-3 in cytoplasm of skin tissue. Compared with NS group, the skin tissue of mice in the DNCB group showed decreased expression of T-bet (Figure 6B) and increased expression of GATA3 (Figure 6C). A-AHT increased T-bet expression and decreased GATA3 expression compared with the DNCB group (Figure 6B, C). The ratio of T-bet/GATA3 was increased in the A-AHT group. These differences were significant in all expressions except the expression of GATA3 (Figure 6C).

\section{Discussion}

Atopic dermatitis (AD), also known as Atopic eczema, is a common chronic and relapsing inflammatory skin disease induced by hapten and mediated by $T$ cells [18]. Clinically the main characteristics of $A D$ are erythema, edema, papule, blister, bleb, bullous reaction and even necrosis. Autohemotherapy (or autologous blood injection), is a treatment using repeated intramuscular injections of AWB to cure certain 
diseases. It has been used in European countries since the end of 20th century [8]. A clinical study has shown that autohemotherapy might be an effective and potentially disease-modifying or curable treatment in patients with AD [9]. In China, autohemotherapy has attracted the interest from many TCM physicians and researchers. Based on the traditional Chinese medicine theory, a new treatment was created by combining the autohemotherapy with the acupuncture therapy, and named as AWB acupoints injection or A-AHT [10]. Acupoints autohemotherapy is considered a more effective therapy with no or few side effects. However, the mechanism is still unclear.

Most of the mechanistic studies on AD point to the imbalance of Th1 and Th2 responses in favor of Th2 responses [19-21]. Other reports indicate that the acute and chronic phases of $A D$ are predominantly a Th2 response and Th1 response, respectively $[2,22]$. IgE is a key downstream biomarker of TH2 cell activation. IgE binds to the high-affinity IgE receptor ( $F c \varepsilon R I)$ found on basophils and mast cells, and the crosslinking of IgE on these cells leads to cellular activation and the degranulation of several inflammatory mediators, such as histamine, thus amplifying the type 2 response. [23]. Mast cell, as one of granular leukocytes, can release many cytokines to mediate inflammatory reaction and immune regulation $[24,25]$. The infiltration of mast cell which was activated by $\operatorname{lgE}$ is one of the key features of AD-like skin. Cytokines released from activated mast cells attract eosinophils into the skin which give rise to skin tissue damage [26]. In this study, an AD-like skin lesions mouse model was established by topical application of DNCB. DNCB-induced AD model in BALB/c mice has been proposed as an appropriate representative of human $A D$ because of similar symptoms including skin erosion, hemorrhage, epidermal hyperplasia, mast cell infiltration and increased IgE level in serum etc. We found that A-AHT reduced the level of serum IgE. At the same time, it effectively alleviated the eczematous symptoms of AD mice, which lays the groundwork for clinical application. Histopathological analysis also revealed infiltration of inflammatory cells, and marked thickening of the epidermis and dermis. Either type of repeated AWB injection (into acupoint or sham acupoint) reduced the severity of these symptoms, suggesting the beneficial effect of AWB injection against AD. According to TB staining slides, the mast cells infiltration in skin tissue of AD model mice were increased by application of DNCB and were decreased remarkably in three treatment groups. The results indicated that all of these treatments have beneficial effects on suppression of mast cell accumulation in DNCB induced AD mice.

Then we focus on the effect of A-AHT about Th1/Th2. First of all, the spleen index of DNCB-induced AD model mice was increased significantly which were markedly reduced after all of the treatments for 35 days. We further assessed the expressions of Th1/Th2 in spleen by flow cytometry because the complex systemic immune reaction of $A D$ was mainly taken place in spleen. The proportion of Th2 cell changes showed that A-AHT regulated Th1/Th2 shift. T cells are the main source of IL-4 and IFN- $\gamma$ which are the representative cytokines about Th1 cells and Th2 cells respectively. Then we detected the levels of IL-4 and IFN- $y$ to roughly speculate Th1 and Th2 differentiation. The results showed that the level of IL-4 and IFN- $y$ increased in the AD model. All of the three treatments had the similar inhibitory effect on levels of IL-4 and upregulation on the ratio of IL-4/IFN- $\gamma$, while differed on Th1/Th2 ratio as A-AHT regulates the body's Th1/Th2 shift. We then identified this function by detecting the related transcription factors of Th1 cells and Th2 cells $[27,28]$. Consistent with its effects on serum IL-4 and IFN-y levels, A-AHT treatment 
increased T-bet expression, and upregulated T-bet/GATA3 ratio compared with the DNCB group. These differences were significant only in A-AHT group. These findings clearly demonstrate that A-AHT treatment inversely affects T-bet and GATA3 expression and IL-4 and IFN-y production, suggesting that the therapeutic efficacy of $A-A H T$ in $A D$ is mediated through modulation of Th1/Th2 responses.

We believe that blood components and acupoint areas are primarily related to anti-inflammation and immune regulatory mechanisms. It is hypothesized that inflammation reaction at the acupoints location triggers local immune response, and after the repeated treatment, the antigenic determinants might be exposed and recognized by body. Thus, the patient's autologous blood is considered as a personalized combination vaccination that stimulates the organism's immune response to the components involved in AE by increasing neutralizing modulators, inducing immune tolerance and re-establishing a new homeostasis [29, 30]. The role of acupoints is demonstrated by the fact that in rats and dogs, acupoint vaccination induced a higher antibody response than in other anatomical sites [31]. Recent studies further provide a neuroanatomical basis for the selectivity and specificity of acupoints in driving the vagal-adrenal anti-inflammatory axis [32]. In this study, we also provide the experimental evidence for the presence of acupoint selectivity (for example, ST36 and IL11 versus non-effective traditional nonacupoint in $5 \mathrm{~mm}$ above ST36 and at the midpoint of the acromial part of the deltoid muscle) and the injectant selectivity (for example, autologous whole blood versus normal saline) in regulating Th1/Th2 immune responses.

\section{Conclusion}

In conclusion, the present study demonstrates that A-AHT attenuates the development of DNCB-induced AD-like skin lesions in BALB/c mouse by inhibiting the level of serum IgE, IL-4 and IFN-ץ, regulating Th1/Th2 shift, and inversely affecting T-bet and GATA3 expression. These results strongly indicate that AAHT could be active potential therapeutic candidate for the prevention and treatment of AD via modulation of both Th1 and Th2 responses.

\section{Abbreviations}

A-AHT: $\quad$ Acupoint autohemotherapy

AD: $\quad$ Atopic dermatitis

ANOVA: $\quad$ Analysis of variance

AOD: $\quad$ Average optical density

AWB: $\quad$ Autologous whole blood

ELISA: Enzyme linked immunosorbent assay

ETFAD: European $A D$ research group 
H\&E: Hematoxylin and eosin

IFN: Interferon

Ig: Immunoglobulin

IHC: Immunohistochemistry

IL: Interleukin

LCs: lymphocytes

TB: $\quad$ Toluidine blue

TCM: Traditional Chinese medicine

Th: $\quad$ Thelper

NBF: $\quad$ Neutral buffered formalin

SEMs: $\quad$ Standard errors of the means

\section{Declarations}

\section{Ethics approval and consent to participate}

All animal experiments were performed according to the Health Guide for the Care and Use of Laboratory Animals and were approved by the medical ethics committee of Southern Medical University.

\section{Consent for publication}

Not applicable

\section{Availability of data and materials}

The datasets used and/or analyzed during the current study are available from the corresponding author upon reasonable request.

\section{Competing interests}

The authors declare that they have no competing interests.

\section{Funding}

The study was supported by a grant from the National Natural Science Foundation of China [grant number 81774390] and the Traditional Chinese Medicine Bureau of Guangdong Province [grant numbers 20201022, 20211258]. 


\section{Authors' contribution}

ZZ and DZ designed the hypotheses and the experiments. ZZ and JH performed the experiments. All authors participated in data interpretation and manuscript review. ZZ was responsible for preparation of the tables and figures and manuscript writing. All authors contributed to the scientific discussion of the data and of the manuscript.

\section{Acknowledgements}

Not applicable

\section{References}

1. Rizk P, Rodenas M, De Benedetto A. Allergen Immunotherapy and Atopic Dermatitis: the Good, the Bad, and the Unknown. Curr Allergy Asthma Rep. 2019,19(12):57. doi:10.1007/s11882-019-0893-z

2. Bieber T. Atopic dermatitis. Ann Dermatol. 2010,22(2):125-137. doi:10.5021/ad.2010.22.2.125

3. Guttman-Yassky E, Krueger JG, Lebwohl MG. Systemic immune mechanisms in atopic dermatitis and psoriasis with implications for treatment. Exp Dermatol. 2018,27(4):409-417. doi:10.1111/exd.13336

4. Mizutani N, Sae-Wong C, Kangsanant S, Nabe T, Yoshino S. Thymic stromal lymphopoietin-induced interleukin-17A is involved in the development of IgE-mediated atopic dermatitis-like skin lesions in mice. Immunology. 2015,146(4):568-581. doi:10.1111/imm.12528

5. Malik K, Heitmiller KD, Czarnowicki T. An Update on the Pathophysiology of Atopic Dermatitis. Dermatol Clin. 2017,35(3):317-326. doi:10.1016/j.det.2017.02.006

6. de Kouchkovsky DA, Ghosh S, Rothlin CV. Negative Regulation of Type 2 Immunity. Trends Immunol. 2017,38(3):154-167. doi:10.1016/j.it.2016.12.002

7. Osinka K, Dumycz K, Kwiek B, Feleszko W. Novel Therapeutic Approaches to Atopic Dermatitis. Arch Immunol Ther Exp (Warsz). 2018,66(3):171-181. doi:10.1007/s00005-017-0487-1

8. Schäfer T. Complementary and alternative medicine (CAM) and atopic eczema. Allergol Select. 2017,1(1):44-52. doi:10.5414/ALX01287E

9. Pittler MH, Armstrong NC, Cox A, Collier PM, Hart A, Ernst E. Randomized, double-blind, placebocontrolled trial of autologous blood therapy for atopic dermatitis. Br J Dermatol. 2003,148(2):307313. doi:10.1046/j.1365-2133.2003.04921.x

10. Huang $C$, Chen $C$, Zhou R, et al. A systematic review and meta-analysis of acupoint autohemotherapy and western medicine therapy in treating chronic obstructive pulmonary disease. Complement Ther Clin Pract. 2021,43:101336. doi:10.1016/j.ctcp.2021.101336

11. Demers G, Griffin G, De Vroey G, Haywood JR, Zurlo J, Bédard M. Animal research. Harmonization of animal care and use guidance. Science. 2006,312(5774):700-701. doi:10.1126/science.1124036 
12. Yang HR, Lee H, Kim JH, et al. Therapeutic Effect of Rumex japonicus Houtt. on DNCB-Induced Atopic Dermatitis-Like Skin Lesions in Balb/c Mice and Human Keratinocyte HaCaT Cells. Nutrients. 2019,11(3):573. doi:10.3390/nu11030573

13. Park JY, Park HJ, Choi YY, Kim MH, Kim SN, Yang WM. Effects of Acupuncture on 1-Chloro-2,4dinitrochlorobenzene-Induced Atopic Dermatitis. Evid Based Complement Alternat Med. 2013,2013:831539. doi:10.1155/2013/982095

14. Wang Z, Yi T, Long M, Ding F, Ouyang L, Chen Z. Involvement of the Negative Feedback of IL-33 Signaling in the Anti-Inflammatory Effect of Electro-acupuncture on Allergic Contact Dermatitis via Targeting MicroRNA-155 in Mast Cells. Inflammation. 2018,41(3):859-869. doi:10.1007/s10753-0180740-8

15. Meng X, Guo X, Zhang J, et al. Acupuncture on ST36, CV4 and KI1 Suppresses the Progression of Methionine- and Choline-Deficient Diet-Induced Nonalcoholic Fatty Liver Disease in Mice. Metabolites. 2019,9(12):299. doi:10.3390/metabo9120299

16. Tsai KS, Chen YH, Chen HY, et al. Antipruritic effect of cold stimulation at the Quchi acupoint (LI11) in mice. BMC Complement Altern Med. 2014,14:341. doi:10.1186/1472-6882-14-341

17. Severity scoring of atopic dermatitis: the SCORAD index. Consensus Report of the European Task Force on Atopic Dermatitis. Dermatology. 1993,186(1):23-31. doi:10.1159/000247298

18. Wang X, Li S, Liu J, et al. Ameliorative effects of sea buckthorn oil on DNCB induced atopic dermatitis model mice via regulation the balance of Th1/Th2. BMC Complement Med Ther. 2020,20(1):263. doi:10.1186/s12906-020-02997-2

19. Galli SJ, Tsai M. IgE and mast cells in allergic disease. Nat Med. 2012,18(5):693-704. doi:10.1038/nm.2755

20. Kim H, Kim JR, Kang H, et al. 7,8,4'-Trihydroxyisoflavone attenuates DNCB-induced atopic dermatitislike symptoms in NC/Nga mice. PLoS One. 2014,9(8):e104938. doi:10.1371/journal.pone.0104938

21. Choi JH, Jin SW, Park BH, et al. Cultivated ginseng inhibits 2,4-dinitrochlorobenzene- induced atopic dermatitis-like skin lesions in NC/Nga mice and TNF-a/IFN- $y$-induced TARC activation in HaCaT cells. Food Chem Toxicol. 2013,56:195-203. doi:10.1016/j.fct.2013.02.037

22. Oyoshi MK, He R, Kumar L, Yoon J, Geha RS. Cellular and molecular mechanisms in atopic dermatitis. Adv Immunol. 2009,102:135-226. doi:10.1016/S0065-2776(09)01203-6

23. Gandhi NA, Bennett BL, Graham NM, Pirozzi G, Stahl N, Yancopoulos GD. Targeting key proximal drivers of type 2 inflammation in disease. Nat Rev Drug Discov. 2016,15(1):35-50. doi:10.1038/nrd4624

24. Krystel-Whittemore M, Dileepan KN, Wood JG. Mast Cell: A Multi-Functional Master Cell. Front Immunol. 2016,6:620. doi:10.3389/fimmu.2015.00620

25. Liu FT, Goodarzi H, Chen HY. IgE, mast cells, and eosinophils in atopic dermatitis. Clin Rev Allergy Immunol. 2011,41(3):298-310. doi:10.1007/s12016-011-8252-4

26. Damask CC, Ryan MW, Casale TB, et al. Targeted Molecular Therapies in Allergy and Rhinology. Otolaryngol Head Neck Surg. 2021,164(1_suppl):S1-S21. doi:10.1177/0194599820965233 
27. Szabo SJ, Kim ST, Costa GL, Zhang X, Fathman CG, Glimcher LH. Pillars article: A novel transcription factor, T-bet, directs Th1 lineage commitment. Cell. 2000. 100: 655-669. J Immunol. 2015,194(7):2961-2975.

28. Hong JH, Kim SH, Lee YC. The Ethanol Extract of Holotrichia diomphalia Larvae, Containing Fatty acids and Amino acids, Exerts Anti-Asthmatic Effects through Inhibition of the GATA-3/Th2 Signaling Pathway in Asthmatic Mice. Molecules. 2019,24(5):852. doi:10.3390/molecules24050852

29. Yong C, Yongpu L, Sufen B, Yao T, Fujuan Q, Changhong X. What happened in the acupoint area of mouse after blood injection? J South Med Univ. 2020,40(9):1225-1229. doi:10.12122/j.issn.16734254.2020.09.01

30. Nahm DH, Ye YM, Shin YS, et al. Efficacy, Safety, and Immunomodulatory Effect of the Intramuscular Administration of Autologous Total Immunoglobulin G for Atopic Dermatitis: A Randomized Clinical Trial. Allergy Asthma Immunol Res. 2020,12(6):949-963. doi:10.4168/aair.2020.12.6.949

31. Jin $H, X u Y$, Shi F, Hu S. Vaccination at different anatomic sites induces different levels of the immune responses. Res Vet Sci. 2019,122:50-55. doi:10.1016/j.rvsc.2018.11.005

32. Liu S, Wang Z, Su Y, et al. A neuroanatomical basis for electroacupuncture to drive the vagal-adrenal axis. Nature. 2021,598(7882):641-645. doi:10.1038/s41586-021-04001-4

\section{Figures}


A

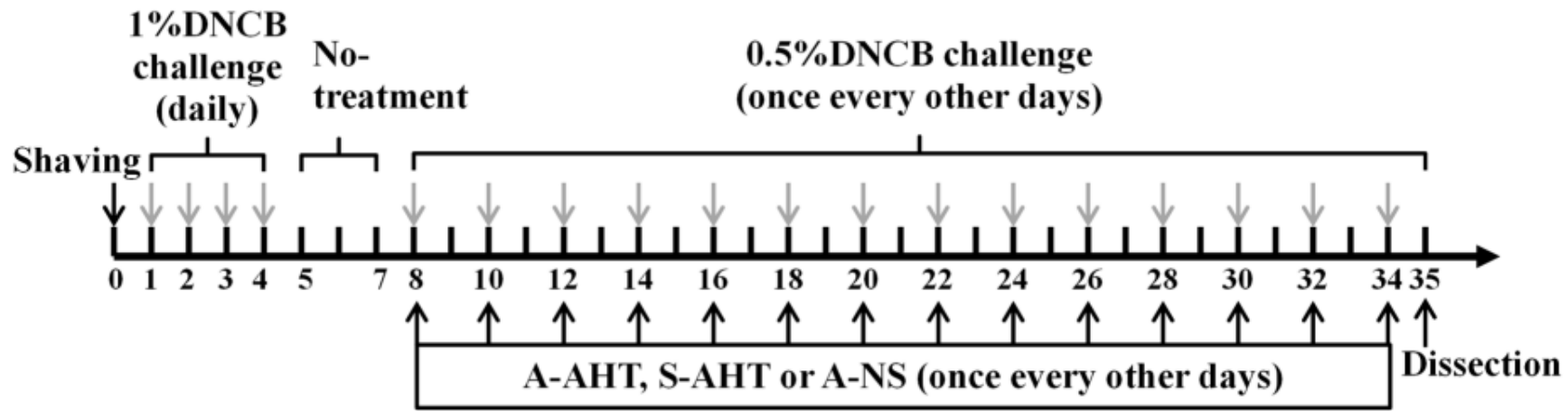

B
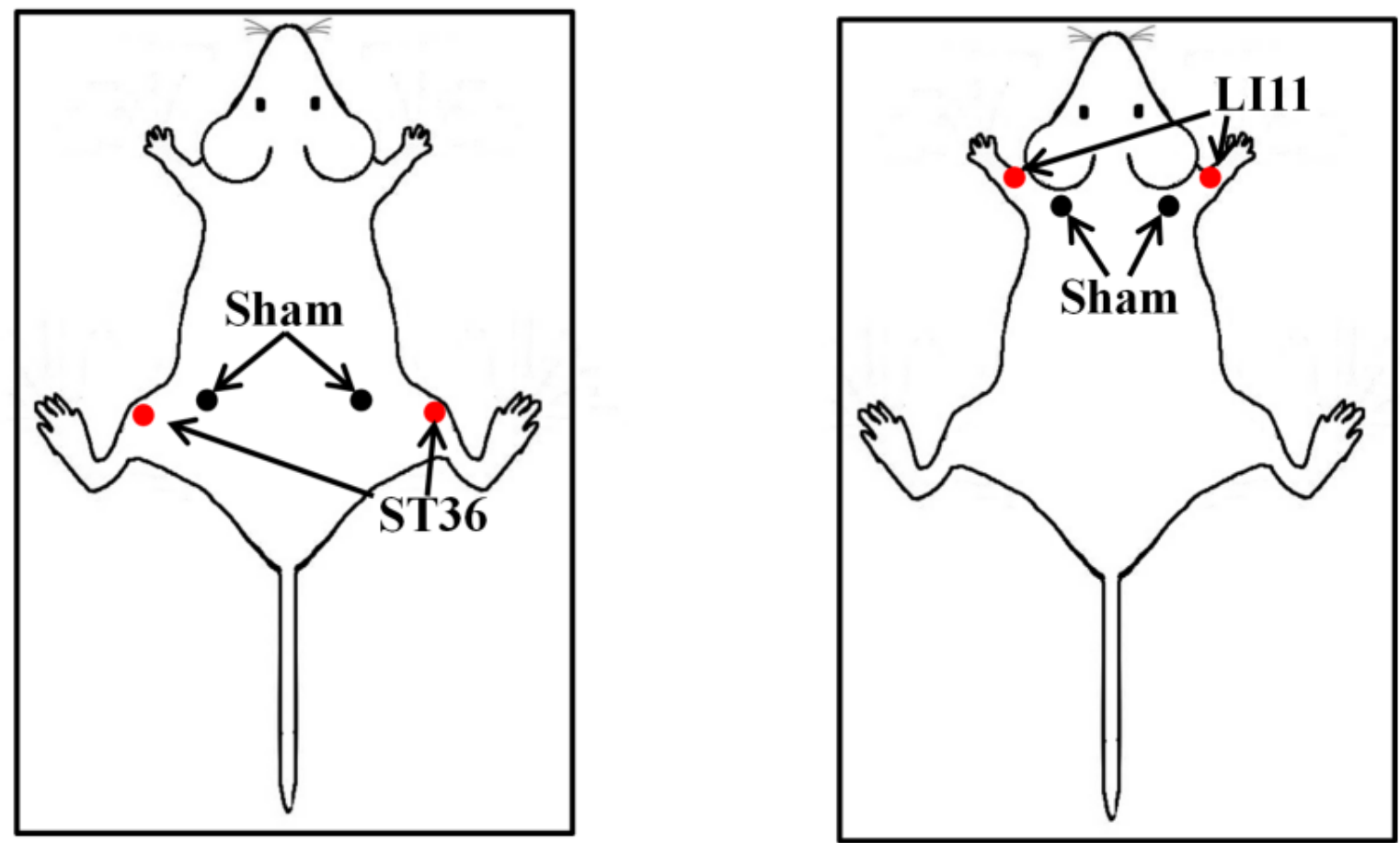

Figure 1

(A) The experimental procedure. (B) The location of ST36, IL11 and sham points. 
A

NC

DNCB

A-AHT

N-AHT

A-NS
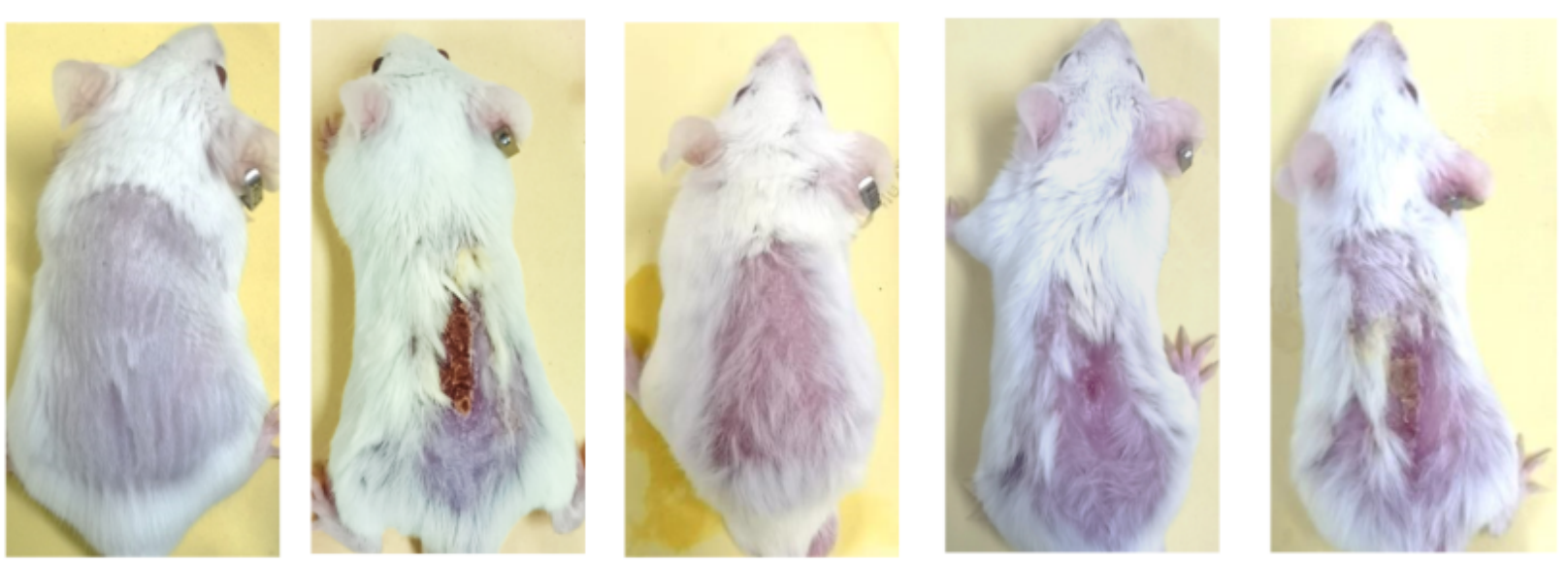

B

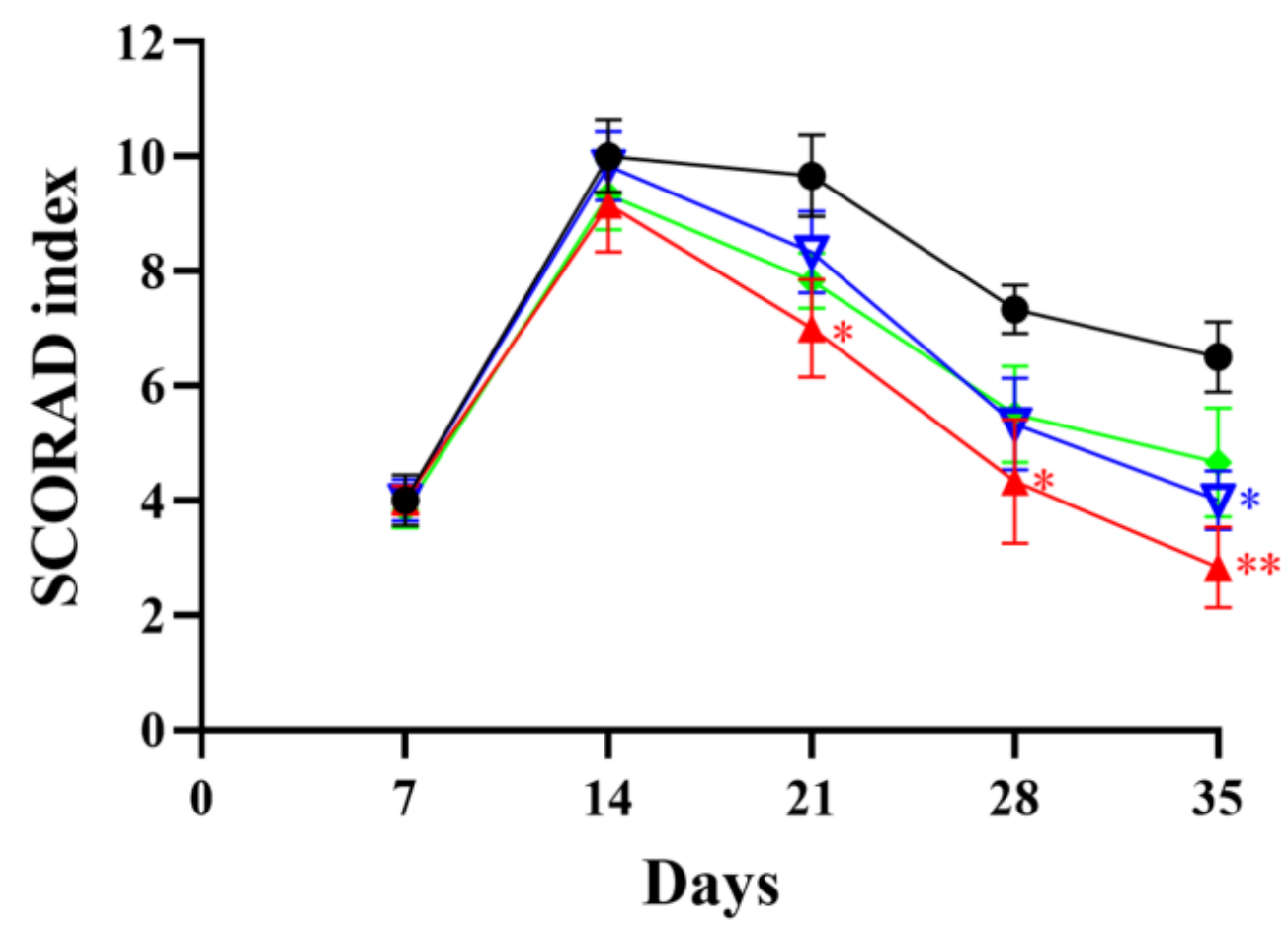

$\rightarrow$ DNCB

$\neg$ A-AHT

$\rightarrow$ N-AHT

$\multimap$ A-NS

\section{Figure 2}

(A) Clinical features of AD-like skin lesions. (B) SCORAD index was measured once a week. SCORAD index were summed according to the six signs (erythema, papule/oedema, oozing/crust, exfoliation, dryness and lichenification). Data were expressed as means \pm SEM ( $n=6$ in each group). Data were analyzed using one-way ANOVA. In $B,{ }^{\star} p<0.05$ and ${ }^{* \star} p<0.01$, compared with DNCB group. 


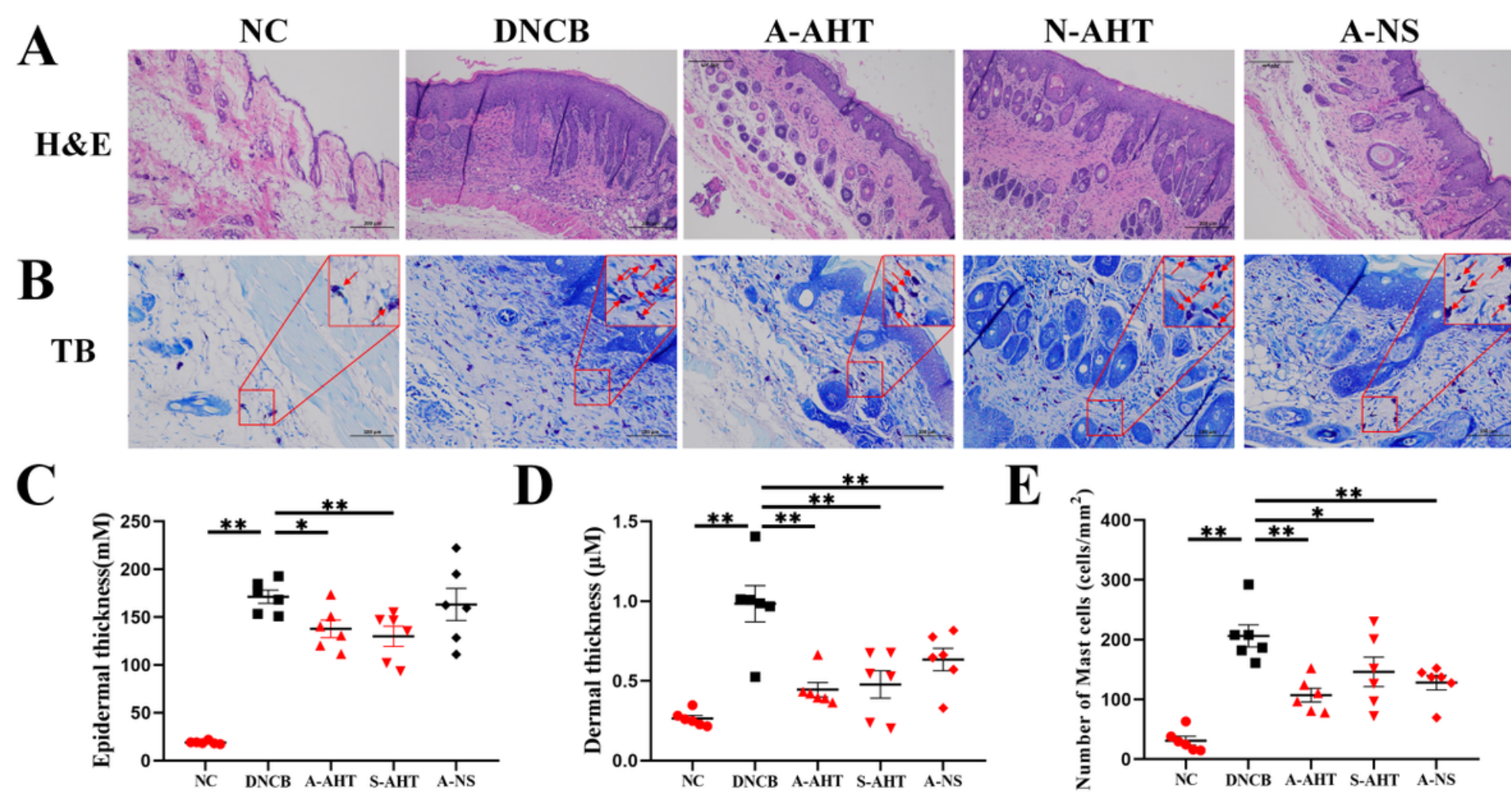

Figure 3

The effects of acupoint autohemotherapy on histopathological features of mice with AD-like skin lesions. (A) The representative image of hematoxylin and eosin (H\&E). The magnification is 100x. (B) Representative images of toluidine blue-stained mast cells in dorsal skin. The magnification is 200x. The typical mast cell infiltrations are indicated by arrows. (C) Measurement of epidermal thickness, (D) dermal thickness and $(E)$ number of mast cells in three fields of each section from all experimental mice. The Data were expressed as means \pm SEM ( $n=6$ in each group). Data were analyzed using one-way ANOVA. In $C-E,{ }^{*} p<0.05$ and ${ }^{* *} p<0.01$ vs DNCB group. 
A $_{\mathrm{NC}}$

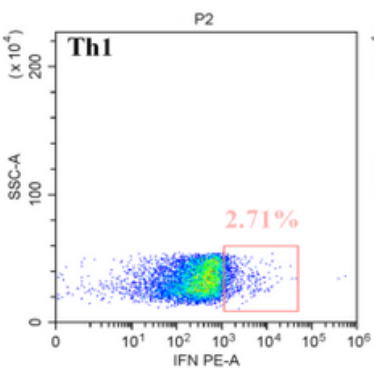

A-AHT

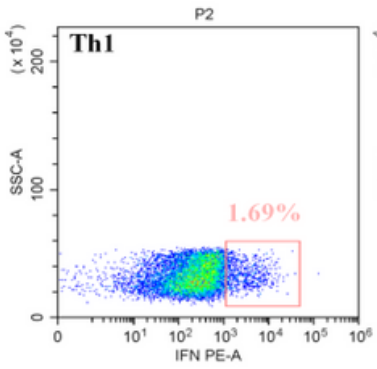

A-NS

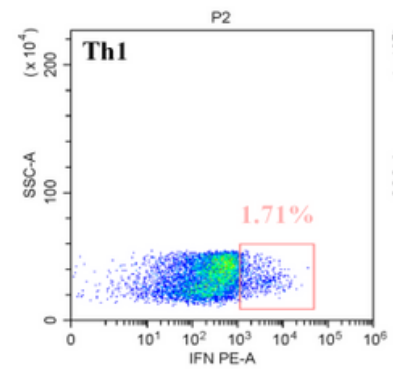

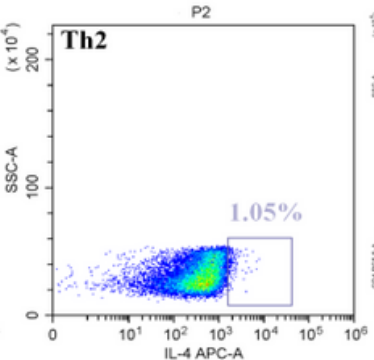

P2

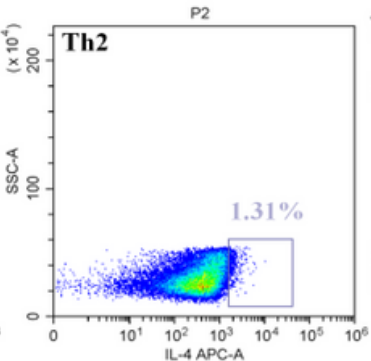

P2

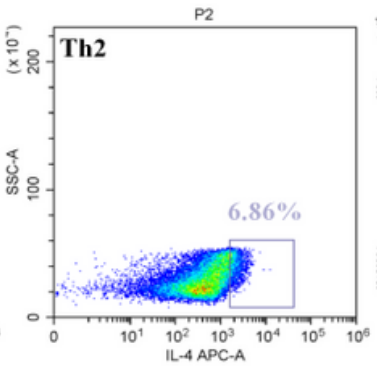

DNCB
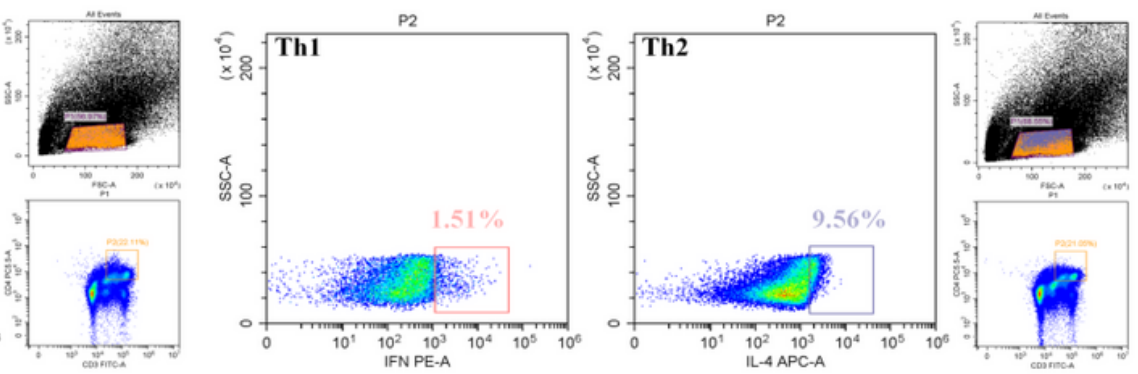

\section{N-AHT}

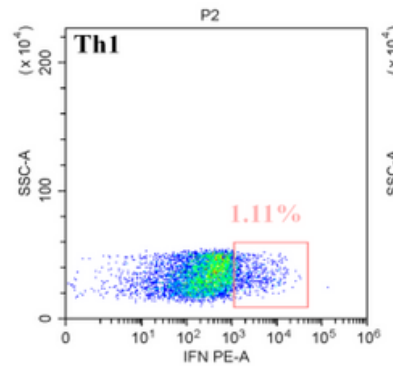

B

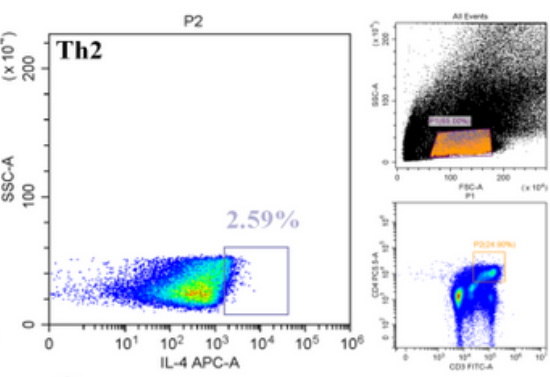

C

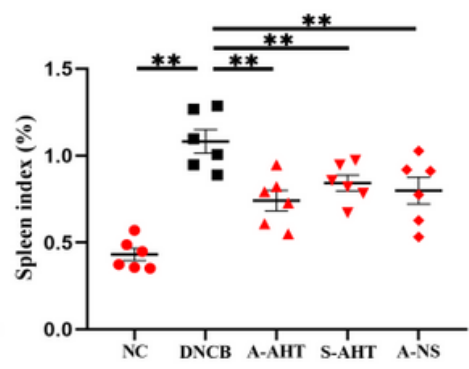

Figure 4

Effects of Acupoint autohemotherapy on Th1/Th2 ratio expression profiling and spleen index in DNCBinduced Balb/c mice. (A) Flow cytometric profiles of Th1 and Th2 cells in murine spleen. (B) Th1/Th2 ratio. (C) Spleen index. Th1 cells were assessed using anti-CD3 $\varepsilon$ FITC, anti-CD4 PerCP-Cy5.5 and anti-IFNy PE. Th2 cells were assessed using anti-CD3 $\varepsilon$ FITC, anti-CD4 PerCP-Cy5.5 and anti-IL-4 APC. Data were expressed as means \pm SEM ( $n=6$ in each group). Data were analyzed using one-way ANOVA. In $B-C,{ }^{*}<$ 0.05 and ${ }^{* *} p<0.01$ vs DNCB group. 
A

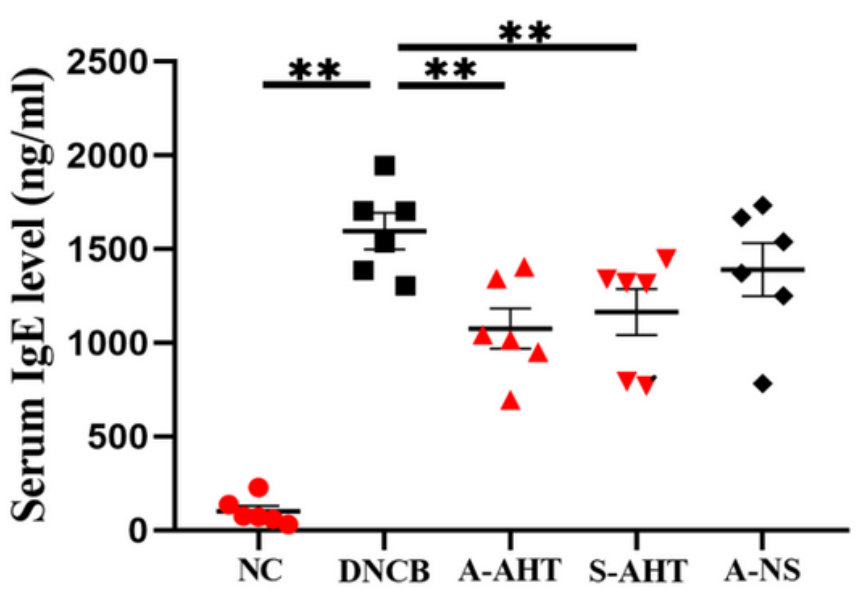

C

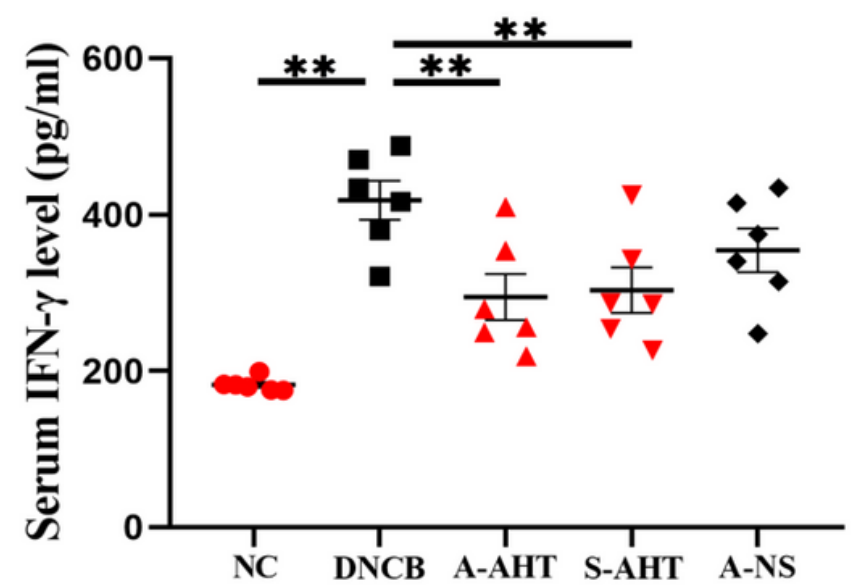

B

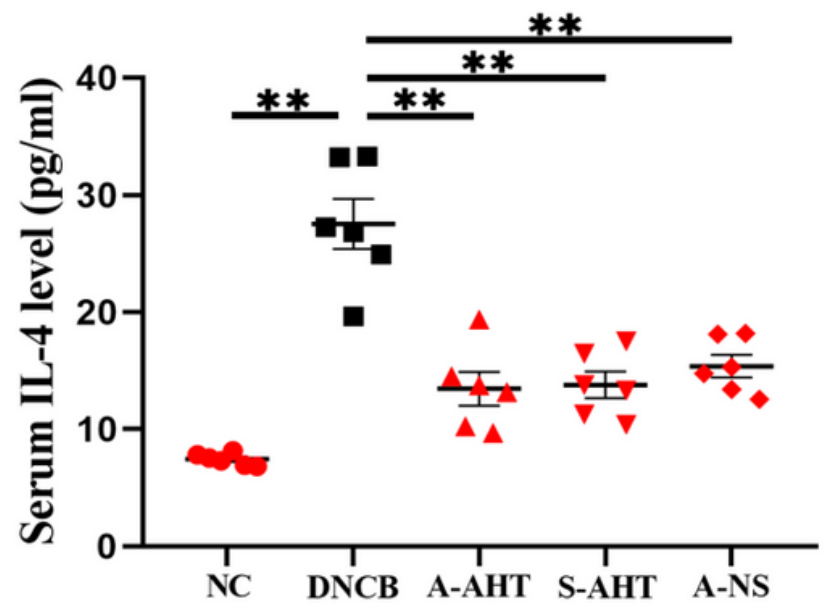

D

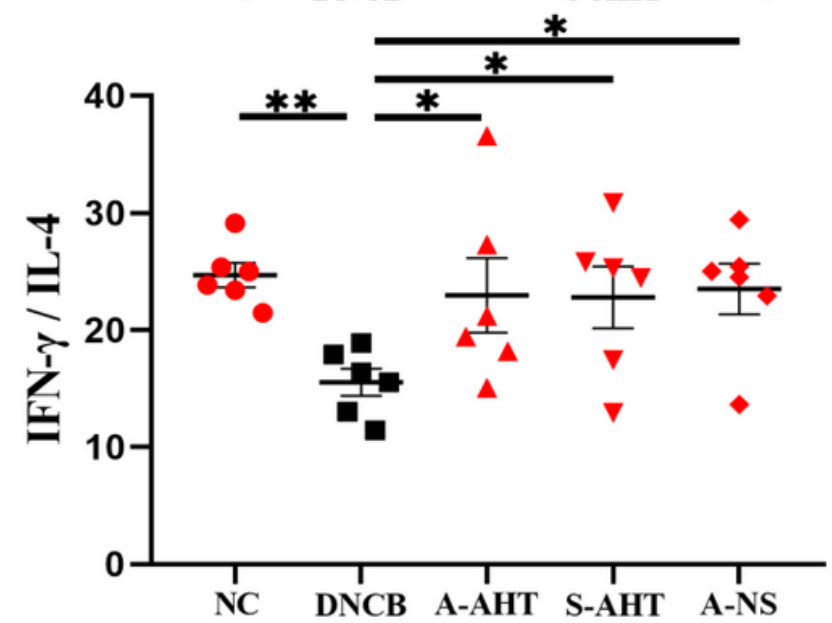

Figure 5

Variations of IgE, IL-4, IFN- $\gamma$ and IFN- - / IL-4 in each group. (A) The level of IgE, (B) IL-4 and (C) IFN- $\gamma$ in serum. (D) The ratio of IFN- $y$ and IL-4. Data were expressed as means \pm SEM $(n=6)$. Data were analyzed using one-way ANOVA. * $p<0.05$ and ${ }^{*} p<0.01$ vs DNCB group. 


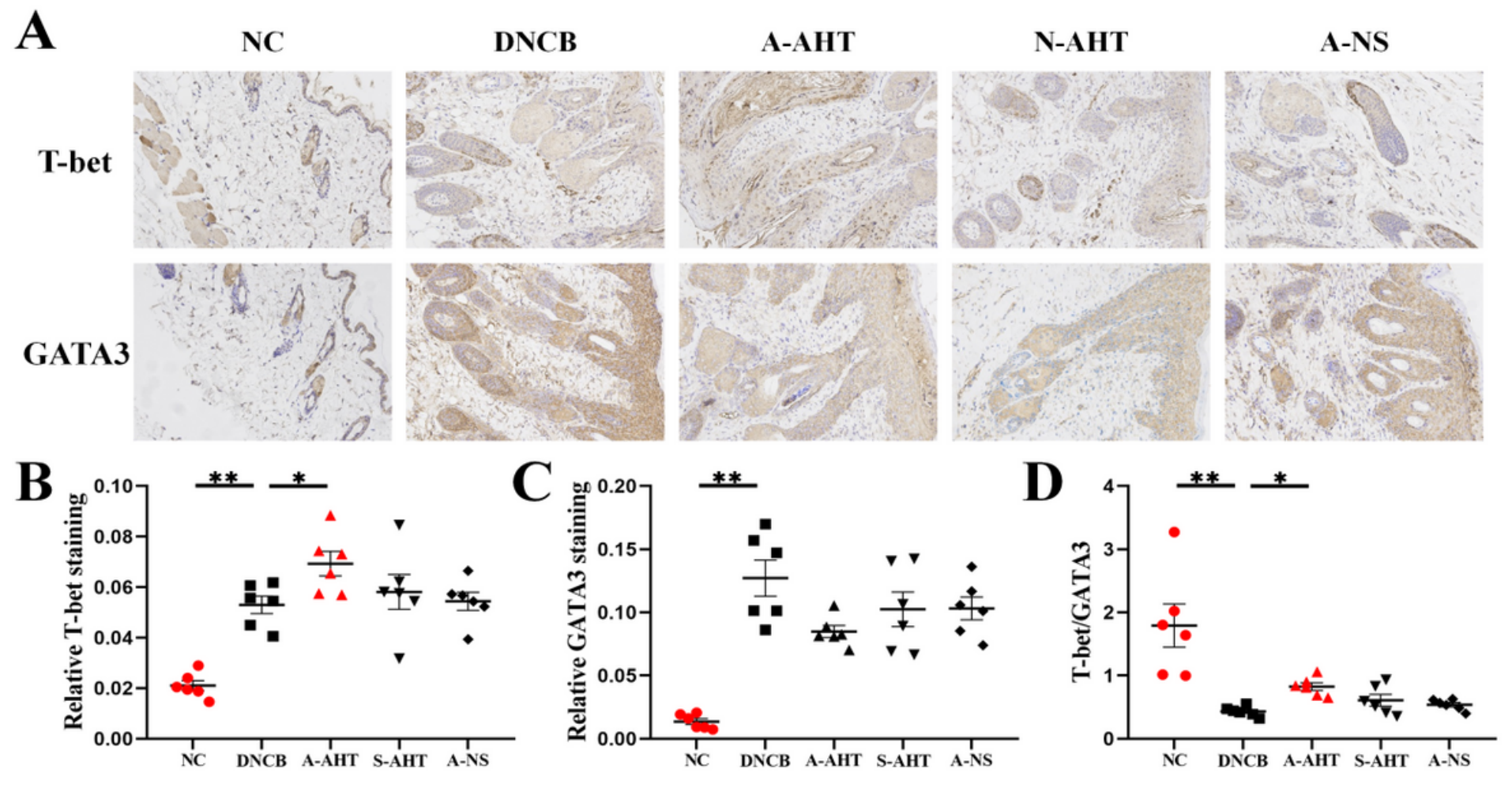

Figure 6

Effects of acupoint autohemotherapy on T-bet and GATA3 expression in skin tissues of each group. (A) Representative images of T-bet and GATA-3. Expression of T-bet and GATA-3 in each group (200x). (B) AOD analysis of T-bet and (C) GATA-3. (D)The ratio of T-bet/GATA-3. Data were expressed as means \pm SEM $(n=6)$. Data were analyzed using one-way ANOVA. In $B-C,{ }^{*} p<0.05,{ }^{\star *} p<0.01$ vs DNCB group. 